Service social

\title{
Évaluateurs, chercheurs et travailleurs sociaux
}

\section{Ricardo B. Zúñiga}

Volume 35, numéro 1-2, 1986

Recherche - Action - Évaluation

URI : https://id.erudit.org/iderudit/706292ar

DOI : https://doi.org/10.7202/706292ar

Aller au sommaire du numéro

Éditeur(s)

École de service social de l'Université Laval

ISSN

1708-1734 (numérique)

Découvrir la revue

Citer cet article

Zúñiga, R. B. (1986). Évaluateurs, chercheurs et travailleurs sociaux. Service social, 35(1-2), 15-32. https://doi.org/10.7202/706292ar d'utilisation que vous pouvez consulter en ligne.

https://apropos.erudit.org/fr/usagers/politique-dutilisation/ 
ZÚNIIGA, Ricardo B., professeur à l'École de service social de l'Université de Montréal.

\section{Évaluateurs, chercheurs et travailleurs sociaux}

\section{Ricardo B. Zúñiga}

La recherche évaluative est un champ en croissance rapide et elle préoccupe les responsables des services sociaux, des programmes sociaux hors-réseau, et de tous ceux qui y travaillent en tant que professionnels ou bénévoles. Les travailleurs sociaux sont eux aussi conscients du rôle de l'évaluation dans leur pratique professionnelle d'intervenants et de fonctionnaires, tout comme de l'importance de comprendre la logique qui la régit.

Mais la recherche évaluative est loin d'être un objet bien défini ou une méthode univoque. Une littérature très abondante de manuels et de décorticages méthodiques - les manuels How to... - proposent une procédure relativement standardisée, apparemment fondée sur le modèle de la recherche expérimentale ou de la planification par objectifs. Bon nombre d'études empiriques qui en découlent situent la recherche évaluative parmi les outils de gestion, et comme une méthode de production de suggestions pour la prise de décisions administratives concernant l'attribution de ressources. Moins connus des services sociaux, les champs de l'éducation et de l'intervention communautaire ont produit toute une autre littérature sur l'évaluation. Si la première prend comme cible l'efficacité des programmes, la deuxième est plus centrée sur la transformation des acteurs au cours de l'actualisation d'un programme donné. La relation entre les deux définit l'ampleur de l'enjeu théorique et la difficulté d'établir une vision assez large du domaine qui permettrait d'éviter sa restriction par des considérations purement idéologiques.

Le présent article a pour but de clarifier le champ conceptuel de la recherche évaluative du point de vue du service social, en soulignant l'ampleur réelle de ce champ, pour mieux situer la place que le 
travailleur social peut et doit y prendre, ainsi que les exigences de formation qui en découlent.

\section{Les concepts d'évaluation}

\section{Une tradition dominante}

Les définitions de l'évaluation semblent se rejoindre, avec peu de différences. Kosecoff et Fink (1982: 15) définissent l'évaluation comme "un ensemble de procédures pour juger les mérites d'un programme et fournir une information sur ses buts, ses attentes, ses résultats prévus et imprévus, son impact et ses coûts». Elle est "le processus par lequel on délimite, obtient et fournit des informations utiles permettant de juger des décisions possibles " (Stufflebeam et al., 1980 : xxvi), « un avis sur l'efficacité d'un traitement ou d'un plan qui a été essayé" (Deming, 1983: 91).

La recherche évaluative, quant à elle, est "l'utilisation systématique des procédures de recherche sociale dans l'estimation de la conceptualisation et du devis, de la mise en œuvre et de l'utilité des programmes d'intervention sociale" (Rossi et Freeman, 1982: 20), avec une conscience confuse d'avoir un mandat de fournir des renseignements qui aideraient à la définition des politiques, sans cependant remplacer les responsables dans la prise de décisions (Brewer, 1983: 16).

L'image assez unifiée de l'entreprise évaluative est centrée sur une conception du "programme » comme une action collective, planifiée à l'avance, avec une rationalité explicite qui prévoit les résultats à atteindre, et qui organise les activités comme des moyens pour atteindre l'objectif préétabli. Compte tenu de cette logique rigoureuse fins/moyens et de cette confiance en une causalité efficace, l'évaluation devient l'évidence empirique de l'atteinte des objectifs et l'analyse de la contribution relative de toute activité et de tout investissement de ressources à l'atteinte de ces objectifs. Le caractère de rationalité instrumentale de l'entreprise permet le développement d'indicateurs et de mesures qui rendent possible la planification dans ce que les publications du Secrétariat d'État appellent "le cadre logique" de la planification, de l'évaluation des projets, et de l'organisation des actions sociales (Canada, Secrétariat d'État, 1983a, 1983b, 1983c). L'évaluation est ainsi consacrée comme étant l'application de la structure même de la rationalité à l'action sociale et comme l'outil privilégié de "rationalisation", détachée de toute option idéologique ou méthodologique autre que celle de la raison elle-même. 


\section{Un complément dissonant}

Cet accord n'est cependant pas sans nuances. Sous une homogénéité dominante, la réflexion sur l'évaluation a aussi mis en évidence les limites du modèle théorique précédent. D'abord, la formulation des objectifs est, en soi, un problème de cohérence entre une action concrète et un discours qui essaie de la décrire, et la différence de ces deux ordres de réalité explique le fait que la formulation verbale ne soit pas nécessairement un reflet exact de l'action qu'elle prétend décrire. Quand la description est en plus celle d'une réalité future, la difficulté est accrue par les limites de l'imagination du "concret futur", du concret qui n'existe pas encore autrement que comme idée. Les stratégies d'évaluation "formative" reflètent la conscience qu'ont les évaluateurs d'un besoin souvent rencontré dans leur pratique, soit de travailler avec les artisans d'un programme pour clarifier et rendre plus explicite sa structure rationnelle, pour accroître son "évaluabilité ".

L'étape de réalisation d'un programme ajoute une autre difficulté : il devient évident que le programme entraîne des objectifs secondaires ou dérivés, et que sa mise en œuvre produit des effets "annexes", "secondaires", "imprévus" - ou même "pervers": la création de dépendances étant l'exemple classique d'effet pervers des thérapies psychologiques et pharmacologiques. Cette constatation déclenche un retour critique sur la stratégie méthodologique de formulation préalable d'objectifs anticipés parce que voulus, et sur ses coûts : celui de négliger l'observation attentive des effets réels d'une action, avec la perte d'éléments imprévus importants, et le coût additionnel d'oublier les effets négatifs, les effets pervers.

Scriven (1974) a proposé de contrer ces risques par le biais d'une solution radicale: que l'évaluation ne démarre pas selon la stratégie traditionnelle consistant à obtenir la définition la plus explicite et la plus consensuelle possible des objectifs préétablis du programme pour essayer de déterminer ensuite le degré dans lequel ils ont été atteints, mais qu'elle démarre plutôt par le simple constat d'effets du programme. En ne commençant pas par l'enquête sur les objectifs, on peut diminuer le risque de la "vision en tunnel». Le discours sur les objectifs est trop souvent déconnecté des objectifs réels et des résultats produits; pourquoi essayer de les traiter séparément? Les projets évoluent dans le temps, et les formulations des objectifs sont vite oubliées.

Et si les résultats sont si souvent différents des objectifs (ils ne les atteignent pas ou ils les dépassent), pourquoi dépenser du temps en essayant de limiter l'évaluation à une mesure de la différence entre 
l'avant et l'après, selon des critères devenus désuets dans l'évolution du projet? II faudrait cependant rappeler que Scriven insiste sur "l'évaluation sans objectifs", et non pas sur "la planification sans objectifs " : il ne veut aucunement diminuer la réflexion préalable sur le sens et l'orientation d'un projet de programme. Son intuition est peutêtre que la planification doit respecter l'autonomie des participants et reconnaître l'émergence du nouveau imprévu ; aussi, dans le processus de l'évaluation, l'autonomie critique de l'évaluateur, dans son observation, est un facteur plus important pour lui aider à comprendre l'activité qu'il est en train d'étudier que sa fidélité à un mandat préétabli. La contribution de Scriven rejoint des courants plus centrés sur l'effort de cerner l'impact de l'innovation et les émergences imprévues, qui sont des effets plus réels que le discours d'intention préalable, et plus larges que les effets attendus (Parlett, 1977).

Une troisième difficulté, sur laquelle nous reviendrons plus tard, s'enracine dans le caractère collectif d'un programme, où les objectifs se présentent comme un discours unique, reflet d'un acteur unique ; il est rare que tel soit le cas. Si le projet est une entreprise participative et consensuelle, ce consensus est difficilement parfait ou statique : ceux qui se sont ralliés au vote majoritaire pourront exercer plus tard leur résistance à la définition accordée, ou exprimer dans leur action leur réinterprétation des objectifs. Si le projet est une entreprise hiérarchique, les acteurs individuels ou collectifs se sentiront légitimés dans la recherche de l'action qui répondrait formellement aux exigences de l'autorité, tout en étant inspirée de leur vision alternative de la logique du projet : un comportement "docile» qui cache une intention "subversive".

\section{La tentation dichotomique}

"L'exposé qui suit s'axe sur certains grands concepts épistémologiques irréconciliables" (Patton, 1980: 20). Quand il s'agit de nuancer l'emprise d'un modèle préétabli de production d'effets, il est facile de percevoir cette nuance comme une alternative méthodologique et même épistémologique. Pour Patton, les critiques au cadre logique sont le résultat inévitable des sources alternatives de connaissance : la méthodologie qualitative, la phénoménologie, l'interaction symbolique, la psychologie de la Gestalt, l'éthnométhodologie, l'école de la Verstehen. 
On peut ainsi construire deux perspectives :

- celle du programme comme objectivité, comme production de transformations du monde vérifiables. Dans cette perspective, la matérialité du programme (activités observables, rendements mesurables) mène à privilégier la logique budgétaire comme source fondamentale d'indicateurs et à concentrer, en conséquence, l'attention sur l'allocation de ressources et sur leur productivité relative; et

- celle des acteurs du programme, des clients, des employés et des gestionnaires. Dans cette perspective, la subjectivité radicale d'une entreprise humaine est une donnée primaire dans sa compréhension, et les constats de transformation ne peuvent pas se limiter à ceux d'une activité observable, même sociale, mais doivent inclure un processus concomitant d'autoproduction des acteurs impliqués. Dans ce processus, leur apprentissage et leur croissance sont un produit fondamental du projet - et un produit difficilement prévisible dans la planification initiale.

Lecomte va encore plus loin et présente les deux perspectives en termes de paradigmes kuhniens concurrentiels: un paradigme normatif, holistique, idéaliste, subjectiviste et informaliste, et un paradigme empirique, élémentaliste, réaliste, objectiviste et formaliste (Lecomte, 1982: 5). L'utilisation du modèle de conflit paradigmatique présente cependant plusieurs problèmes, le plus fondamental étant celui formulé par Kuhn lui-même comme celui de l'incommensurabilité : il n'y a pas un point de vue supra-paradigmatique à partir duquel les paradigmes puissent être comparés. Cette discussion, périphérique au sujet, me pousserait vers la tentation de suggérer qu'une telle comparaison peut être concevable seulement à partir du paradigme empiriste : cette déconnection du fait et de la valeur est inacceptable pour le premier des deux paradigmes.

À un niveau plus pratique, nous pourrions nous poser des questions sur les conséquences de l'habitude épistémologique en service social selon laquelle toute distinction est formulée en termes de dichotomies qui forcent un choix entre des perspectives que cette stratégie intellectuelle a converties en objets réels autonomisés: la théorie et la pratique, l'individuel et le social, la personne et l'environnement, le qualitatif et le quantitatif. Un vocabulaire relationnel ou dialectique ne réussit pas à neutraliser les coûts sociaux de la transformation d'une distinction en une séparation, pour employer une terminologie aristotélicienne. Dans une opposition d'exclusion mutuelle, une fois celle-ci acceptée comme telle, la seule 
prise de position cohérente est celle de "choisir son camp", et construire un discours d'opposition, qui inclut le panégyrique d'un camp et le dénigrement de l'autre.

Le sens du travail de Lecomte reste néanmoins valable: "Le problème est de savoir combiner la validité de la question avec la validité de la réponse... " (Lecomte, 1982 : 15). Le problème, et sur lequel nous reviendrons dans la conclusion, est celui de dépasser la pensée dichotomique, la forme épistémologique la plus primitive de tenir compte des différences, pour chercher des modèles qui placent comme fondement de la logique de l'évaluation une conception du social " en émergence continue, et plus que simplement rationnel ", qui confronte l'unité d'une conception intellectuelle à la multiplicité réelle des acteurs, des intérêts et des perspectives (Cochran, 1980).

\section{L'évaluation : rationalité et utilisation}

\section{Une utilisation sociétale}

Dans un premier moment de l'analyse, on peut dire du champ de l'évaluation en général ce que Scriven dit de la recherche évaluative : elle "doit produire comme conclusion ce que les chercheurs sociaux ont appris pendant des années de formation à considérer illégitime : un jugement de valeur, de qualité, de mérite" (Scriven, 1974: 4). L'évaluation se trouve trop souvent déchirée entre deux discours : celui de l'objectivité, de la connaissance approfondie et systématique des faits qui est la récompense de l'impartialité (les commissions d'enquête, les "recherches évaluatives»), et celui de l'incorporation à la prise de décisions, "objectives" bien sûr, et basées seulement sur des faits (l'évaluateur-arbitre), mais aussi efficaces du point de vue du gestionnaire (l'évaluation des coûts pour découvrir "le gras", la résolution de conflits de travail, la proposition d'alternatives organisationnelles, la « rationalisation » des activités et des services). De fait, l'évaluation incarne la tension interne de son discours dans une variété de rôles difficilement compatibles: l'auto-évaluation du professionnel responsable, le rôle de l'agent de programme dans la conception, l'analyse, l'actualisation et l'évaluation de programmes, celui du chercheur subventionné et celui du consultant contractuel en gestion.

L'évaluation de programmes et la recherche évaluative se confondent et s'unifient comme outils de gestion. "La fonction première de la recherche évaluative dans une organisation est d'aider à 
la prise de décision " (Bergeron, 1985 : 22). "Subventions à la recherche en évaluation, [...] pour la réalisation des recherches appliquées reliées à l'évaluation des programmes..." (Québec, Ministère des affaires sociales, 1985). Pour les uns, "l'évaluation et la recherche scientifique ne poursuivent pas tout à fait les mêmes fins. [...] II ne s'agit pas d'une conception opposant l'évaluation et la recherche, mais plutôt d'une façon de voir l'évaluation comme étroitement liée à la prise de décision et à l'action" (Beaudoin, 1982: 155); pour les autres, l'évaluation et la recherche évaluative peuvent être différenciées par à peu près toutes leurs dimensions signifiantes (voir Tableau 1).

\section{TABLEAU 1}

\section{Comparaison de la recherche et de l'évaluation.}

\begin{tabular}{|l|l|l|}
\hline & \multicolumn{1}{|c|}{ Recherche } & \multicolumn{1}{c|}{ Evaluation } \\
\hline $\begin{array}{l}\text { Motivation de } \\
\text { l'investigateur }\end{array}$ & Curiosité scientifique & Solution d'un problème \\
\hline $\begin{array}{l}\text { Objectifs de } \\
\text { l'investigation }\end{array}$ & Conclusions & Décisions \\
\hline $\begin{array}{l}\text { Rôle de } \\
\text { l'explication }\end{array}$ & Explications : lois, vérité & Descriptions : valeur \\
\hline $\begin{array}{l}\text { Autonomie de } \\
\text { l'investigateur }\end{array}$ & Grande & Faible \\
\hline $\begin{array}{l}\text { Utilité sociale } \\
\text { des découvertes }\end{array}$ & Indirecte & Directe \\
\hline $\begin{array}{l}\text { Généralisation } \\
\text { des résultats }\end{array}$ & Grande & Faible \\
\hline $\begin{array}{l}\text { Contrôle } \\
\text { expérimental }\end{array}$ & Essentiel & Possible \\
\hline $\begin{array}{l}\text { Critères pour } \\
\text { juger l'activité }\end{array}$ & Validité interne & Isomorphisme \\
\hline $\begin{array}{l}\text { Base discipli- } \\
\text { naire }\end{array}$ & Unidisciplinaire & Multidisciplinaire \\
\hline
\end{tabular}

Source: M.-A. Nadeau, 1981 : 55. 
II y a un déblayage à faire dans l'utilisation des concepts d'évaluation de programmes et de recherche évaluative. Leur utilisation comme synonymes prête à confusion et rend difficile une analyse des fonctions de l'évaluation. Pour Rafter (1984), la recherche évaluative peut avoir trois buts :

- un but scientifique, relié à la vérification hypothético-déductive des postulats;

- un but interactif, qui est celui d'alimenter les acteurs par l'analyse conjointe du problème, la génération des données, l'analyse et la décision conjointe ;

- un but hybride, en deux étapes, dans lequel l'expert fournit de l'information aux preneurs de décisions pour ensuite décider avec eux de l'utilisation de l'information. Pour Schön, Drake et Miller (1984), il faut penser à une " réflexion-dans-l'action », dans laquelle la stratégie d'intervention peut être modifiée selon les résultats obtenus dans les premières étapes, avec une utilisation des données qualitatives pour aider à la discrimination parmi des hypothèses alternatives. Pour eux, l'expérimentation aurait en conséquence trois fonctions : exploration, pour provoquer une réponse et apprendre ainsi quelque chose de nouveau ; mise à l'épreuve d'une intervention, de son efficacité, mais en prenant en considération l'ensemble des résultats prévus et imprévus, voulus et non voulus; mise à l'épreuve d'une hypothèse, pour discriminer sa valeur relative parmi d'autres.

Ces analyses permettent de revenir à la critique fondamentale de Gauthier (1982), sur la stérilité scientifique de la plupart des "recherches» évaluatives, dans le degré où leur rationalité ne va pas plus loin que les intérêts des commanditaires d'évaluer l'utilisation "rationnelle" des ressources.

La confusion quant à la différence des buts d'une évaluation de programme et d'une recherche évaluative n'est pas un problème découlant purement des théories différentes de la science. La modalité d'évaluation des demandes de subvention à la recherche commence à prendre une forme inquiétante : elle sépare et déconnecte les critères de "pertinence " (correspondance aux priorités politiques de l'organisme subventionnaire) de ceux de "qualité" (adhésion à "la méthode scientifique", comprise selon la version positiviste), et réduit le champ du scientifique à cette "qualité ". Comme exemple de cette tendance, les renseignements sur les subventions de recherche de la Direction de l'Évaluation des programmes du Ministère des affaires sociales indiquent que la demande "est analysée par un comité interne qui en évalue la pertinence en fonction de son degré de priorité ", et que " tous 
les projets de recherche sont soumis à une évaluation de qualité par un comité de pairs".

La rationalité institutionnelle qui détermine les priorités du fonctionnement des services est sans doute légitime ; mais la recherche scientifique peut-elle se passer d'une rationalité à elle, de ses propres priorités? La formation de chercheurs ne doit-elle pas se refléter dans la détermination scientifique des priorités de recherche? Si on reprend la formule de Lecomte, qui doit garantir la validité de la question? Les coûts politiques d'une restriction de la rationalité scientifique à "la méthode" sont clairs :

"Le rôle du chercheur comme un consultant plutôt que comme un acteur autonome, directeur de son action à lui, maximise la manipulation de croyances dans la recherche. Une structure de pouvoir avec les ressources pour engager des chercheurs en sciences sociales appliquées, pour se servir de la machinerie des sciences sociales et pour utiliser à son bénéfice la dissémination des résultats produit un biais injuste en faveur du statu quo et dans la production de croyances apparemment dérivées des sciences sociales appliquées. " (Campbell, 1982: 135).

\section{Une conception de la connaissance}

Trois postulats épistémologiques semblent fonder les conceptions de la recherche évaluative :

- l'action évaluée doit démontrer une vision claire et anticipée des résultats qu'elle prévoyait atteindre. Le concept organisateur est celui d'objectif, et il se définit comme "pré-vision", préfiguration du résultat, différent dans cette spécificité matérielle des concepts comme ceux de mission, de but et de finalité, qui peuvent rester davantage reliés à des valeurs ou à des intentions;

- elle suppose une théorie prédictive, une connaissance empirique préalable qui permet de postuler que l'action qui a été planifiée et qui sera entreprise aura comme effet le résultat désiré, que les moyens choisis se sont avérés efficaces pour mener à la fin préétablie; et

- elle suppose une capacité de contrôle de la situation, un pouvoir d'assurer que les moyens nécessaires pour faire l'évaluation soient disponibles, et une autorité de gestion suffisante pour que les actions prônées par l'évaluation puissent être mises en œuvre. 
Ces postulats ne peuvent être apodictiques. Le service social n'a pas développé une tradition de débat épistémologique, et ce sousdéveloppement peut être à la base de beaucoup de ses problèmes d'incohérence et de perte de pouvoir décisionnel. Les postulats précédents lient une conception de la connaissance à une conception du pouvoir politique, et acceptent comme corollaire la dévalorisation intellectuelle des acteurs qui ont un pouvoir politique restreint. Non seulement ils laissent sans réponse des questions fondamentales dans la construction des connaissances (qui sont ceux qui apprennent? quel impact peuvent-il avoir dans la modification du programme? quelle capacité a le programme d'apprendre de ceux qui sont dans la première ligne de sa mise en œuvre, et donc de tenir compte d'une réalité qui peut être autre que celle qui était présupposée à l'avance ?), mais ils ne permettent pas d'apprendre de l'action et ils sont, en conséquence et en dernière instance, antiscientifiques (tout en étant parfaitement rationnels.)

Le modèle épistémologique dominant de la recherche évaluative ne dépasse pas l'opposition entre une "formation", qui devrait être le produit d'une formation en sciences sociales et qui fournirait la démarche, la "méthode", et une "pratique", qui ne serait que l'application déductive de cette démarche à une expérience d'intervention et à une réalité de travail, qui ne fournirait donc que le problème-cible. La " théorie " enfanterait " la méthodologie d'intervention ", celle-ci enfanterait les "modèles " et les "grilles"... et le praticien "appliquerait" toute cette sagesse reçue à une réalité docile. Si la recherche évaluative est conçue sur le modèle de la stratégie hypothético-déductive, la seule contribution du praticien serait sa compétence formelle dans l'application d'une technologie méthodique, et la contribution des participants et des clients se verrait réduite à celle d'objets de l'évaluation.

\section{Évaluation, services sociaux et service social}

\section{Les acteurs}

La littérature sur l'évaluation a une fâcheuse tendance à utiliser les termes " usager » et " preneur de décisions " comme étant interchangeables. Le concept d'acteur institutionnel élargit davantage le champ des personnes concernées: 
"[...] la législation a identifié un grand nombre d'acteurs plus ou moins autonomes et a réparti les responsabilités et les pouvoirs entre ces acteurs institutionnels, que ce soit les établissements, les conseils régionaux, les conseils de médecins et de dentistes, les syndicats, les corporations professionnelles, le Conseil du trésor, etc. Il y a également d'autres acteurs qui ne sont pas inscrits dans la loi mais qui se sont développés en fonction de diverses légitimités, tels la coalition des malades, les médias, les associations d'établissements, certains acteurs individuels, etc. " (Bergeron, $1985: 33$ ).

Une analyse sociologique part d'un point de vue plus descriptif, plus ouvert, que celui qui cherche uniquement à identifier les preneurs de décisions - trop souvent limités à l'administrateur/commanditaire. La définition des acteurs reste néanmoins la clé de la définition d'un enjeu social, et le travailleur social qui intervient dans une évaluation sans respecter cette analyse réduit sa marge d'autonomie aux buts de son employeur. Le rôle du chercheur s'identifie à celui du consultant organisationnel, et celui de l'usager à celui de client (Mandel, 1984; Lescarbeau, Payette et Saint-Arnaud, 1985).

\section{Les projets, les processus et les programmes}

Pour le travailleur social, le concept même de programme exige une réflexion plus critique que l'acceptation de la rationalité instrumentale véhiculée par le concept. Un programme n'est pas seulement un "cadre logique " de structuration d'une action sociale. II implique aussi le caractère d'une proposition d'action sociétale avec une légitimation et une sanction qui lui donnent le caractère d'action officielle.

Dans ce sens, un programme implique d'abord un contrôle externe, soit un lien à l'appareil politique d'État, même s'il est aussi ténu que l'octroi d'un numéro en tant qu'organisme de charité, et aussi un contrôle interne, qui agit comme répondant et comme responsable du respect des buts et de l'allocation des moyens tels qu'ils ont été autorisés. Le programme inclut ainsi, non seulement une promesse d'efficacité acceptée par une instance externe au programme, mais aussi une promesse de respect du déroulement prévu, de l'allocation des ressources, et du caractère vérifiable du résultat. Un programme est un investissement sociétal et, en tant que tel, il doit joindre la transparence de son action à l'acceptation préalable de sa logique interne par une source de légitimation politique qui lui est extérieure. 
Mais, malgré tous les efforts de contrôle, un programme reste une entreprise humaine, imprévisible dans ses détails, et un champ permanent de négociations :

"Les acteurs ont leur rationalité propre; il y en a plusieurs, ils sont en compétition les uns avec les autres et ils n'ont pas tous la même force politique ou économique. Il ne s'agit pas d'un système fermé, mais d'un ensemble d'interconnections avec divers environnements. Les problèmes ou enjeux ne sont pas bien définis ; ils sont généralement ambigus, les perceptions varient selon les acteurs; ils font appel aux valeurs ou aux droits des individus; ils peuvent également comporter des aspects idéologiques. L'information est au mieux contestée, interprétée, manipulée ou même déformée selon les convictions idéologiques. Quant au temps, il est généralement limité et ceci fait aussi partie de la stratégie des acteurs en présence. " (Bergeron, 1985 :

34.)

À l'intérieur d'un programme, dans le quotidien du déroulement de son processus d'actualisation, le travailleur social est souvent plus que le responsable de l'actualisation; il y est aussi participant, et souvent porteur d'un projet, noyau intentionnel de l'action ou d'une conduite qui se détermine «à la fois par rapport aux facteurs réels et présents qui la conditionnent et par rapport à un certain objet à venir qu'elle tente de faire naître" (Sartre, $1960: 135$ ). Ce projet, qui peut animer une action collective à laquelle le travailleur social s'identifie, unifie dans les meilleurs des cas l'action collective.

Dans les cas les plus déchirants, la conscience professionnelle peut placer le praticien entre sa perception des intérêts des participants ou des clients et la structure concrète du programme dans lequel il travaille (procédures, règlements, structures de décision). Dans ces cas, la recherche d'une base d'évaluation de l'activité confronte le professionnel à la distance qui peut se produire entre les objectifs d'un programme et le sens qu'ils prennent dans son déroulement. C'est dans cet écart que doivent se situer les soucis des professionnels qui perçoivent la distance entre la demande des clients et sa transformation par la logique rigide des programmes (Sherif, 1981) ainsi que les exigences d'assurer des formes d'évaluation des programmes qui donnent une voix aux usagers (Von Schönberg, 1985).

\section{La boîte à outils du travailleur social}

Le sens de cette réflexion sur la richesse changeante de la recherche évaluative était de la relier à l'action du travailleur social et à la formation qui lui est nécessaire. Ce but exige trois réflexions. 


\section{Un contexte théorique déchiré}

Le champ de la recherche évaluative a longtemps ignoré la critique des modèles scientifiques de type positiviste, et elle reste encore avec un certain retard dans son insertion dans le remous paradigmatique actuel des sciences sociales. Rafter signale le fait que, pour des auteurs aussi influents que Carol Weiss, James Q. Wilson et Edward Suchman, "l'approche scientifique représente le modèle normatif pour la recherche évaluative " par sa défense farouche de la validité interne de la recherche. L'échantillonnage représentatif, le devis de type expérimental et l'analyse statistique multivariée restent les critères opérationnels du caractère scientifique, et les exigences de contrôle marginalisent le client à un rôle de fournisseur des réponses (Rafter, 1984: 167-168).

Une telle vision doit faire face à la marée croissante des critiques méthodologiques qui remettent en question la possibilité d'observations théoriquement neutres permettant d'évaluer les effets d'un programme en le confrontant aux résultats d'une expérience elle aussi théoriquement neutre (Cook, Levinson-Rose et Pollard, 1980 : 487), et qui acceptent que la réalité spécifique des systèmes sociaux fait que les évaluations des réformes sociales doivent accepter que ces réformes soient «des processus de changement médiatisés symboliquement, compréhensibles seulement si nous réussissons à comprendre les raisons qui ont guidé l'effort de recherche de solutions pratiques à des problèmes sociaux " (Dunn, 1982: 298). Les problèmes et les variables seraient les construits du chercheur, avec un sens qui évoluerait tout au long de la transaction du chercheur avec la situation dans laquelle il contribuerait à créer les phénomènes instables, uniques et incertains qu'il étudie (Schön, Drake et Miller, 1984: 33).

Et si le service social ne participe pas à ce débat, la conception positiviste continuera à alimenter la rhétorique de l'opposition entre la théorie et la pratique, entre le qualitatif et le quantitatif, et les écoles de service social continueront à répéter leur catéchisme: "hors de la Science, point de salut" - pendant que les travailleurs sociaux continueront à essayer d'agir de façon responsable, réfléchie et critique, mais sans réaliser le potentiel de production de connaissances de cette accumulation expérientielle, faute d'un langage adéquat et pertinent à la réflexion sur l'intervention.

\section{La place de la pensée opérationnelle}

La formation en service social n'a jamais donné à l'analyse par variables la place qu'elle aurait pu avoir si on avait pris au sérieux la 
rhétorique que prônaient l'isomorphisme de la pratique professionnelle et la recherche, telle qu'on la retrouve depuis Mary Richmond jusqu'à nos auteurs américains contemporains (pour une discussion et une remise en question de cette tradition, voir : Zúñiga, 1986). La pensée opérationnelle, la définition en termes d'observations, la construction d'indicateurs, la vérification expérimentale, l'analyse par variables et l'explication par corrélation de variables sont moins présentes dans la littérature professionnelle que dans les sentiments d'infériorité coupable des travailleurs sociaux, qui ne les utilisent pas mais n'osent pas remettre en question le dogme de leur valeur scientifique absolue.

La pratique du service social puise dans d'autres sources. La revendication d'une insertion organique dans le monde des sciences sociales apparaît seulement dans les contextes reliés à la formation, dans les écoles universitaires et dans les congrès. Un niveau plus fréquent de structuration intellectuelle est celui des "modèles de pratique ", dans lequel on formule un discours qui relie des valeurs à des procédures d'intervention, avec un lien vague aux concepts isolés, tirés des sciences sociales. Les modèles définissent les problèmes, identifient les dynamiques sociales ou psychologiques sous-jacentes, et développent surtout les actions qui seraient les plus adéquates compte tenu des présupposés conceptuels.

Mais les pratiques les plus fréquentes représentent une stratégie cognitive qui n'a malheureusement pas reçu l'attention qu'elle mérite. Dans celle-ci, des valeurs semblent se joindre à une expertise acquise dans l'expérience d'intervention et à une confiance du travailleur social dans le réalisme de sa perception et dans sa capacité de trouver, dans son expérience, des actions adéquates aux situations qu'il confronte. Dans cette stratégie, le plan d'intervention ne peut être qu'une esquisse minimale étant donné le caractère transactionnel de l'intervention.

En tant que stratégie cognitive, elle a des forces et des faiblesses qui, toutes deux, sont pertinentes à la prise de position face à l'évaluation. Comme force, l'intervention est guidée par un dialogue constant entre l'orientation générale que le praticien donne à son action et sa perception de l'orientation des personnes avec lesquelles il entre en interaction. Comme faiblesse, les deux perceptions appartiennent, en dernière instance, au praticien, et sont trop souvent moulées par ses intentions, son projet personnel. Si la théorie sartrienne du projet parlait d'un dialogue entre le sujet et l'objet qu'il essaie de faire naître, cette orientation fondée sur les valeurs peut l'appauvrir à un monologue du praticien avec lui-même, à un dialogue entre ses valeurs, qui le guident, et ses perceptions, qui le confirment dans ses convictions. 
L'observation exige une certaine discipline épistémologique, et un effort pour donner à l'objet une certaine autonomie par rapport à l'observateur. Il y a peu dans la tradition intellectuelle du service social pour soutenir cet effort. Une pensée orientée par le désir d'action de transformation du réel, selon un idéal personnel de développement ou de croissance, en est une pour laquelle la résistance au changement peut stimuler une persévération réitérative plutôt qu'une remise en question de l'adéquation des perceptions de la cible d'intervention et des résultats de l'intervention. $\mathrm{Si}$, en plus, une telle pensée est orientée par des valeurs abstraites ou mal formulées (pensons à "la croissance", "la prise en charge", "l'autonomie", "la participation" et "le changement social »), il y a un danger sérieux de rester dans le monde des intentions et refuser les évidences qui pourraient menacer une orientation ou un choix de stratégie: il n'y a pas d'interventions inadéquates, il y a seulement des structures organisationnelles trop rigides et des clients pas assez motivés.

Dans le monde des valeurs, l'exigence fondamentale de l'évaluation, celle d' "être à l'écoute des résultats de mon intervention", apparaît comme crise de foi, hérésie. La recherche évaluative sur l'efficacité de l'action devient une interpellation menaçante, une remise en question de mes motifs. Dans le monde des valeurs éthiques, l'analyse stratégique est suspecte et l'analyse tactique est stigmatisée comme opportuniste. Les religions s'appuient sur leurs convictions non empiriques et ne tirent pas trop de leçons de l'expérience (elles sont même un peu embarrassées d'en tirer); le service social n'a pas de vérité révélée ni de valeurs universelles sur lesquelles appuyer son action, et il ne devrait pas suivre leur exemple.

\section{Une multiplicité irréductible}

L'évolution des conceptions des sciences du social et le réalisme foncier des praticiens peuvent générer une attitude qui rendra l'évaluation et même la recherche évaluative plus proche de leurs intérêts, et peuvent aussi générer le type de prise de conscience sur laquelle ils pourraient fonder une réflexion méthodique. La pensée méthodologique reliée à l'évaluation a insisté depuis longtemps sur le dépassement du scientisme, en soulignant qu'aucun instrument de mesure ne peut isoler un paramètre théorique et que l'imperfection et la contamination éliminent la possibilité pratique des définitions opérationnelles. Le besoin des confirmations multiples affaiblit la valeur de l'entrevue et du questionnaire comme méthodes autosuffisantes, et rend nécessaire un plus grand souci de mesures 
multiples. "Une fois qu'une proposition a été confirmée par deux mesures indépendantes et différentes, l'incertitude de son interprétation diminue de façon significative [...] Si une proposition peut survivre à une série de mesures imparfaites, avec toutes leurs erreurs, il devient plus raisonnable de se fier à elle" (Webb et al., 1981: 3).

La stratégie de "triangulation" (Webb et al., 1981; Denzin, 1978), qui suppose l'utilisation de mesures multiples, valorise l'observation du praticien et le praticien lui-même, parce qu'elle diminue l'importance de l'apport de l'expert externe. Celui-ci se fie trop souvent à un instrument unique de mesure en raison de sa spécialisation personnelle, d'un souci d'économie de temps, et de sa conscience que les commanditaires reconnaissent son expertise et se sentent sécurisés dans l'utilisation d'instruments tels que l'enquête et le questionnaire.

C'est dans la réflexion plus poussée sur le contenu de l'expérience professionnelle que le service social pourrait raffermir les fondements scientifiques de sa pratique, et qu'il pourrait également établir un lien plus organique avec la recherche évaluative dans ce qu'elle représente de respect de la réalité, de respect de l'autre et de respect de la responsabilité d'une action transformatrice réfléchie, démocratique et efficace.

\section{Références bibliographiques}

BeAudoIn, A., "La problématique de l'utilisation de la recherche évaluative", dans: R. LeCOMTE et L. RUTMAN (éds), Introduction aux méthodes de la recherche évaluative, Québec, Les Presses de l'Université Laval, 1982: 155-176.

BERGERON, P., "La recherche évaluative au ministère des Affaires sociales", Carrefour des affaires sociales, vol. 7, no 2, 1985: 33-36.

Brewer, M.B., "Evaluation: past and present", dans: M.B. BreWER et E.L. StRUENING (éds), Handbook of Evaluation Research, Beverly Hills, Sage, $1983: 15-27$.

CAMPBelL, D.T., "Experiments as arguments", Knowledge: Creation, Diffusion, Utilization, vol. 3 n० 3, 1982: 327-337.

CANADA, Secrétariat d'État, Programme des groupes minoritaires de langue officielle, Guide de planification. "L'avenir, ça se planifie». 1983a.

CANADA, Secrétariat d'État, Programme des groupes minoritaires de langue officielle, Guide d'évaluation de projet. "Une évaluation pratique centrée sur l'utilisation ". 1983b.

CANADA, Secrétariat d'État, Programme de promotion de la femme, Région du Québec (Angèle Aubin), Passons à l'action. 1983c. 
Cochran, N., "Society as emergent and more than rational: an essay on the inappropriateness of program evaluation ", Policy Sciences, vol. 12, 1980 : 113-129.

COOK, T.D., J. LEVINSON-ROSE et W.E. POLLARD, "The misutilization of evaluation research : some pitfalls of definition ", Knowledge: Creation, Diffusion, Utilization, vol. 1, no 4, $1980: 477-498$.

Deming, W.E., "The logic of evaluation", dans : M.B. Brewer et E.L. Struening (éds), Handbook of Evaluation Research, Beverly Hills, Sage, 1983 : 91-106.

DENZIN, N.K., The Research Act. A Theoretical Introduction to Sociological Methods, New York, McGraw-Hill, 1978.

DunN, W.N., "Reforms as arguments", Knowledge: Creation, Diffusion, Utilization, vol. 3, no 3, 1982: 293-326.

Gauthier, B., Méta-évaluation en affaires sociales: analyse de cent cas de recherches évaluatives, Québec, Conseil québécois de la recherche sociale, 1982.

KoseCOFf, J. et A. FINK, Evaluation Basics. A Practitioner's Manual, Beverly Hills, Sage, 1982.

LECOMTE, R., "Les paradigmes méthodologiques de la recherche évaluative: leurs fondements et leurs répercussions", dans: R. LECOMTE et L. RUTMAN (éds), Introduction aux méthodes de recherche évaluative, Québec, Les Presses de l'Université Laval, 1982: 1-21.

Lescarbeau, R., M. Payette et Y. St-Arnaud, Devenir consultant. Instrument autogéré d'intervention, 2 vols. Montréal, Les Presses de l'Université de Montréal, 1985.

MANDEL, M.B., "The design and selection of evaluation studies : a user-oriented framework and its assessment", Knowledge: Creation, Diffusion, Utilization, vol. 5, no 4, 1984 : 419-445.

Nadeau, M.-A., L'évaluation des programmes d'études. Théorie et pratique. Québec, Les Presses de l'Université Laval, 1981.

Parlett, M., "Evaluation as illumination: a new approach to the study of innovatory programs", dans: D. HAMILTON (éd.), Beyond the Numbers Game. A Reader in Educational Evaluation, Berkeley, McCutchan, 1977 : 6-22.

PATTON, M.Q., "Un nouveau paradigme de recherche en évaluation", dans: C. Paquette, G.E. Hein et M.Q. Patton, Évaluation et pédagogie ouverte. Esquisse d'un processus évaluatif appliqué aux systèmes ouverts, Victoriaville (Québec), N.H.P., $1980:$ 15-42.

Québec, Ministère des affaires sociales, Liste de documents publiés par la Direction de l'Évaluation des programmes, 1985.

RAFTER, D.O., Three approaches to evaluation research ", Knowledge : Creation, Diffusion, Utilization, vol. 6, no 2, 1984 : 165-185.

Rossi, P.H. et H.E. Freeman, Evaluation. A systematic approach, $2^{\mathrm{e}}$ éd., Beverly Hills, Sage, 1982.

SARTRE, J.-P., Question de méthode, Paris, Gallimard, 1960.

SCHON, D.A., W.D. DraKe et R.I. Miller, "Social experimentation as reflection-inaction: community-level nutrition intervention revisited", Knowledge : Creation, Diffusion, Utilization, vol. 6, no 1. 1984: 5-36. 
SCRIVEN, M., "Evaluation perspectives and procedures ", dans : W.J. POPHAM (éd.), Evaluation in Education. Current Applications, Berkeley, McCutchan, 1974: 3-93.

SHERIF, T., "L'intervention des centres de services sociaux dépend de l'interprétation des demandes qui leur sont faites", dans: Association canadienne des sociologues et anthropologues de langue française, L'intervention sociale. Actes du Colloque 1981, Montréal, Albert SaintMartin, 1982: 35-43.

Stufflebeam, D.I. et al., L'évaluation en éducation et la prise de décision, Victoriaville (Québec), Éditions N.H.P., 1980.

VON SCHÖNBERG, B., Les points de vue des clients et des citoyens : leur place dans l'évaluation des programmes, Québec, Ministère des affaires sociales, Direction de l'évaluation des programmes. 1985. ("Études et analyses ", 19.)

WebB, E.J., D.T. Campbell, R.D. Schwartz, L. SeChrest et J.B. Grove, Nonreactive Measures in the Social Sciences, $2^{\mathrm{e}}$ éd., Boston, Houghton-Mifflin, 1981.

ZúÑIGA, R. (éd.), La recherche évaluative. Guide du cours SVS-6520, Montréal, Université de Montréal, École de service social, 1985, 291p., (polycopié).

ZÚNIGA, R., "Logique de la recherche et logique de l'intervention. Une approche méthodologique au débat sur la recherche en service social", Revue canadienne de service social, 1986 (sous presse). 